\title{
Histone deacetylase inhibitor ITF2357 leads to apoptosis and enhances doxorubicin cytotoxicity in preclinical models of human sarcoma
}

\author{
Marta Di Martile ${ }^{1}$, Marianna Desideri ${ }^{1}$, Maria Grazia Tupone ${ }^{1}$, Simonetta Buglioni ${ }^{2}$, Barbara Antoniani ${ }^{2}$, \\ Carlotta Mastroiorio ${ }^{1}$, Rita Falcioni ${ }^{3}$, Virginia Ferraresi ${ }^{4}$, Nicola Baldini ${ }^{5,6},{ }^{2}$ Roberto Biagini ${ }^{7}$, Michele Milella ${ }^{4}$, \\ Daniela Trisciuoglio (1,8 and Donatella Del Bufalo ${ }^{1}$
}

\begin{abstract}
Sarcomas are rare tumors with generally poor prognosis, for which current therapies have shown limited efficacy. Histone deacetylase inhibitors (HDACi) are emerging anti-tumor agents; however, little is known about their effect in sarcomas. By using established and patient-derived sarcoma cells with different subtypes, we showed that the panHDACi, ITF2357, potently inhibited in vitro survival in a p53-independent manner. ITF2357-mediated cell death implied the activation of mitochondrial apoptosis, as attested by induction of pro-apoptotic $\mathrm{BH} 3$-only proteins and a caspasesdependent mechanism. ITF2357 also induced autophagy, which protected sarcoma cells from apoptotic cell death. ITF2357 activated forkhead box (FOXO) 1 and 3a transcription factors and their downstream target genes, however, silencing of both FOXO1 and 3a did not protect sarcoma cells against ITF2357-induced apoptosis and upregulated FOXO4 and 6. Notably, ITF2357 synergized with Doxorubicin to induce cell death of established and patient-derived sarcoma cells. Furthermore, combination treatment strongly impaired xenograft tumor growth in vivo, when compared to single treatments, suggesting that combination of ITF2357 with Doxorubicin has the potential to enhance sensitization in different preclinical models of sarcoma. Overall, our study highlights the therapeutic potential of ITF2357, alone or in rational combination therapies, for bone and soft tissue sarcomas management.
\end{abstract}

\section{Introduction}

Soft tissue and bone sarcomas are rare and aggressive tumors, including $>50$ histological subtypes with an overall 5 -year survival for all stages of about $55 \%^{1,2}$. Available therapeutic options for sarcoma patients with advanced or metastatic tumors are anthracyclines (Doxorubicin or Epirubicin)-based chemotherapy regimens ${ }^{3-5}$.

Correspondence: Daniela Trisciuoglio (daniela.trisciuoglio@ifo.gov.it) or Donatella Del Bufalo (donatella.delbufalo@ifo.gov.it)

${ }^{1}$ Preclinical Models and New Therapeutic Agents Unit, IRCCS Regina Elena National Cancer Institute, Rome, Italy

${ }^{2}$ Pathology Unit, IRCCS Regina Elena National Cancer Institute, Rome, Italy Full list of author information is available at the end of the article

These authors contributed equally: Marta Di Martile, Marianna Desideri.

These authors jointly supervised this work: Daniela Trisciuoglio, Donatella Del Bufalo.
Despite advances in the understanding of the molecular basis of sarcomas, these are still insufficient for the development of new-targeted strategies to potentially improve the management of localized or advanced sarcomas and the dismal prognosis of patients.

Inhibition of histone deacetylases (HDACs), enzymes regulating chromatin topology and gene expression ${ }^{6}$ are considered as new potential therapeutic agents able to reverse aberrant histone acetylation and gene transcription of cancer cells. HDAC inhibitors (HDACi) have been reported as effective anti-cancer molecules alone or in combination in preclinical $^{7,8}$ and clinical studies ${ }^{9-11}$. In particular, several preclinical studies demonstrated that 


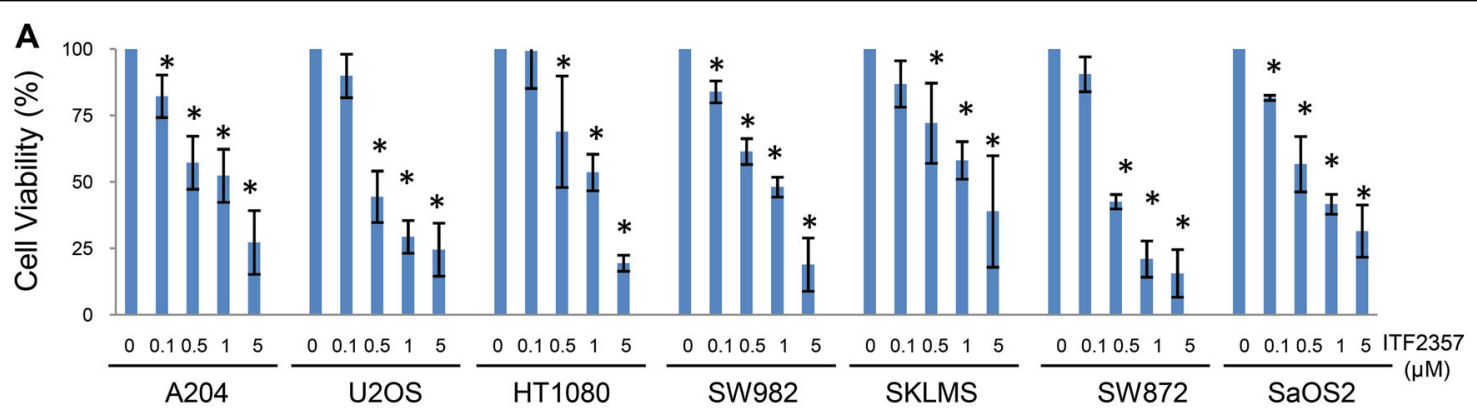

B

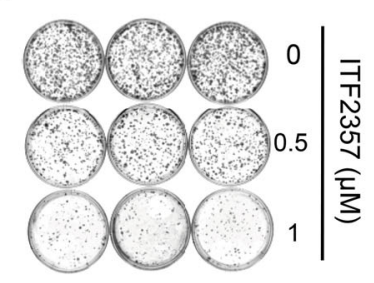

C

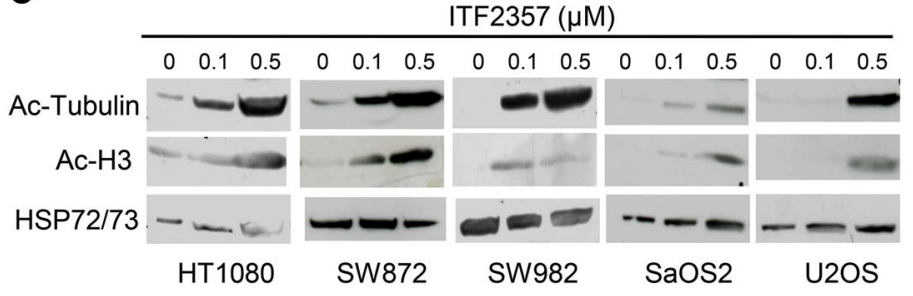

D

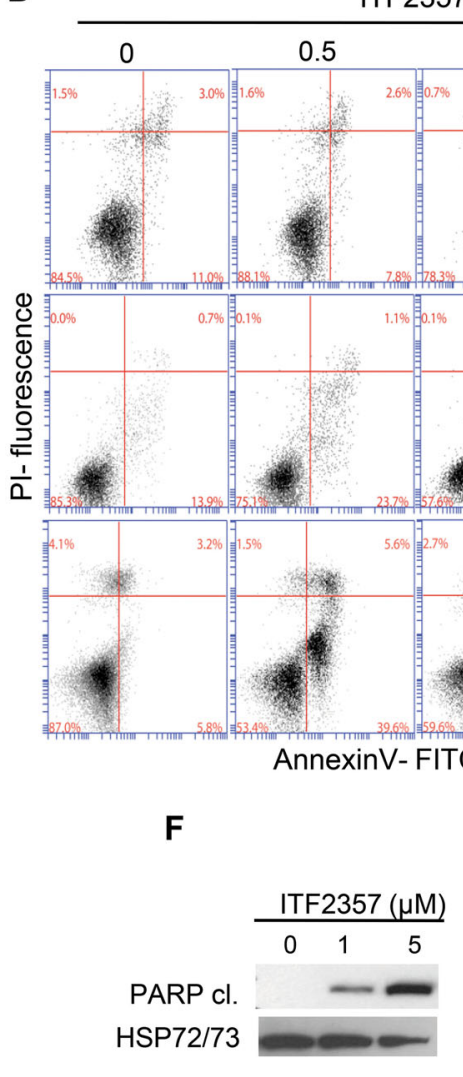

E

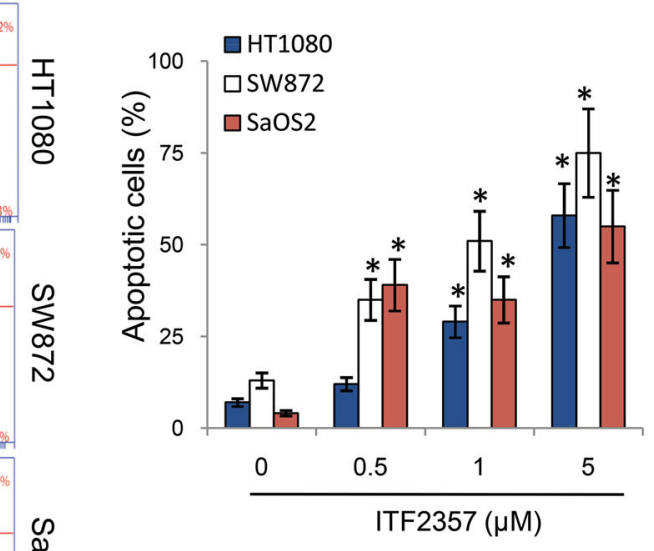

Fig. 1 (See legend on next page.)

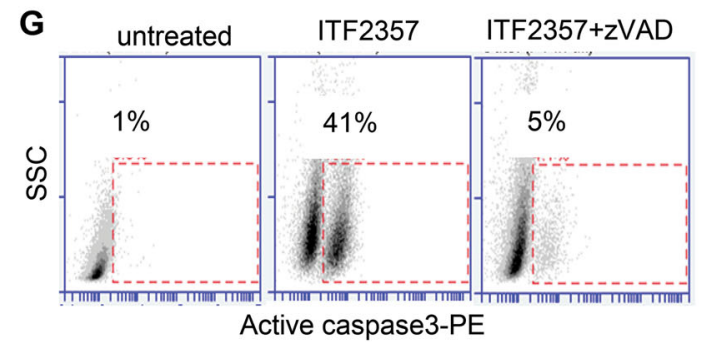


Fig. 1 ITF2357 affects survival of human sarcoma cells. a Analysis of cell viability by MTT assay in the indicated sarcoma cell lines treated for $72 \mathrm{~h}$ with increasing concentrations of ITF2357. The results are reported as "viability of drug-treated cells/viability of control cells" $\times 100$ and represent the mean \pm SD of three independent experiments performed in triplicate. b Colony formation of HT1080 cells treated for $24 \mathrm{~h}$ with increasing concentrations of ITF2357. Representative images are shown. c Western blot analysis of acetylated histone H3 (Ac-H3), acetylated a-Tubulin (AcTubulin) in total cell lysates from the indicated cell lines treated with 0.1 and $0.5 \mu \mathrm{M}$ ITF2357 for $24 \mathrm{~h}$. d Representative experiment of apoptotic cells evaluation by AnnexinV/PI staining in the indicated cell lines exposed to increasing concentration of ITF2357 for $72 \mathrm{~h}$. e Quantification of apoptosis by AnnexinV/PI staining in the indicated cell lines treated for $72 \mathrm{~h}$ with increasing concentrations of ITF2357. The results represent the mean \pm SD of three independent experiments. $\mathbf{f}$ Western blot analysis of cleaved form of PARP (PARP cl.) in total cell lysates from HT1080 cells treated with 1 and 5 $\mu \mathrm{M}$ ITF2357 for $72 \mathrm{~h}$. c, f HSP72/73 expression was used as loading and transferring control. Western blots representative of two independent experiments with similar results are shown. $\mathbf{g}$ Flow cytometric analysis of active caspase-3-PE staining in HT1080 cells untreated or treated for $72 \mathrm{~h}$ with $1 \mu \mathrm{M}$ ITF2357 alone or in combination with $50 \mu \mathrm{M}$ pan-caspase inhibitor zVAD-fmk (ZVAD). A representative experiment out of two is shown. $\mathbf{a}, \mathbf{e}$ $p$ values were calculated between untreated and treated cells, ${ }^{*} p<0.01$

HDACi alone or in combination therapy constitute novel therapeutics versus different sarcoma histotypes including Ewing $^{12,}{ }^{13}$, epithelioid ${ }^{14}$, liposarcoma ${ }^{15}$, synovial and rhabdomyosarcomas ${ }^{16-18}$, and osteosarcoma ${ }^{19-23}$. HDACi activities have been mainly due to their effect on cell growth and survival, on apoptosis or autophagy, or cancer stem cells ${ }^{12,13,15,24-27}$. On the basis of these preclinical evidences, some HDACi against sarcomas have been moving from phase I to phase II clinical trials alone or in combination anthracyclines ${ }^{9,10,28-30}$.

Here, we wanted to evaluate the effect of a newgeneration HDACi, ITF2357 (Givinostat) in human sarcoma cell lines. ITF2357 is a safe and tolerable panHDACi with broad anti-inflammatory properties ${ }^{31,} 32$. ITF2357 anti-tumoral activity has been reported in several hematologic ${ }^{33-35}$ and solid tumor ${ }^{36,37}$ models, however, little is known about ITF2357 activity in sarcomas. In the present study, we investigated the molecular and functional effects of ITF2357 in preclinical models of soft tissue and bone sarcomas. We discovered that targeting HDACs by ITF2357 induces a mitochondrial apoptosis in human sarcoma cells. More importantly, ITF2357 enhances in vitro Doxorubicin (Doxo) cytotoxicity in both established and patient-derived sarcoma cells. Furthermore, combination treatment strongly impaired xenografts tumor growth in vivo, when compared to single treatments.

\section{Results}

ITF2357 reduces in vitro human sarcoma cell growth and induces apoptosis by activating mitochondrial apoptotic pathway

Firstly, we assessed the effect of ITF2357 on cell viability of a panel of established human sarcoma cell lines showing different p53 status, including osteosarcoma (SaOS2, U2OS), liposarcoma (SW872), synovial sarcoma (SW982), fibrosarcoma (HT1080), rhabdomyosarcoma (A204), and leiomyosarcoma (SKLMS), that represent the major histological entities of sarcoma family. As shown in Fig. 1a and Fig. 1 suppl., ITF2357 strongly reduced sarcoma cell viability regardless of p53 status, with IC50 values ranging from $3 \mu \mathrm{M}$ in the p53 null line SaOS2, to 0.57-0.97 $\mu \mathrm{M}$ in p53-mutant lines (SW872, SKLMS) and 0.63-0.88 $\mu \mathrm{M}$ in wild-type (wt) p53 lines (U2OS, SW982, HT1080, A204). To analyze ITF2357 effect on long-term survival of sarcoma cells, we evaluated the clonogenic growth of the representative HT1080 cell line upon ITF2357 treatment. Of note, ITF2357 suppressed colony growth in a dose-dependent manner (Fig. 1b). A 70\% reduction in clonogenic ability was observed after treatment with $1 \mu \mathrm{M}$ ITF2357. Furthermore, exposure to ITF2357 markedly increased histone $\mathrm{H} 3$ and $\alpha$-tubulin protein acetylation in a dose-dependent manner in sarcoma cell lines (Fig. 1c).

By using HT1080, SW872, and SaOS2 representative cell lines, to study in depth molecular mechanisms of ITF2357 we evaluated whether growth inhibition by ITF2357 was due to apoptosis induction. Hence, we assessed the apoptotic effects of ITF2357 by cytofluorimetric analysis of AnnexinV/propidium iodide (PI) staining. A dose-dependent increase in apoptotic cells was observed after treatment with ITF2357 (Fig. 1d, e) in all cell lines even though at different extent. Taken together, these data indicate that ITF2357 treatment inhibited cell proliferation in all sarcoma cell lines tested and that the reduction in the cell viability was associated with induction of apoptosis independently of p53 status.

Focusing our attention on HT1080 cells, we explored whether treatment with ITF2357 induced caspasedependent apoptosis by using the pan-caspase inhibitor, zVAD-fmk (zVAD). As shown in Fig. 1f, ITF2357 triggered the cleavage of poly(ADP ribose) polymerase (PARP), a typical caspases substrate. We found that about $40 \%$ of ITF2357-treated cells were positive for active caspase- 3 staining, whereas $<5 \%$ of zVAD-pretreated cells, as well as, untreated cells were positive for caspase-3 staining (Fig. 1g). In agreement with these results, pharmacological inhibition of caspases protected HT1080 cells against ITF2357-induced loss of cell viability (Fig. 2a suppl.). Next, we analyzed whether ITF2357-induced apoptosis requires the mitochondrial apoptotic pathway. To this aim, we used HT1080 cells overexpressing the 

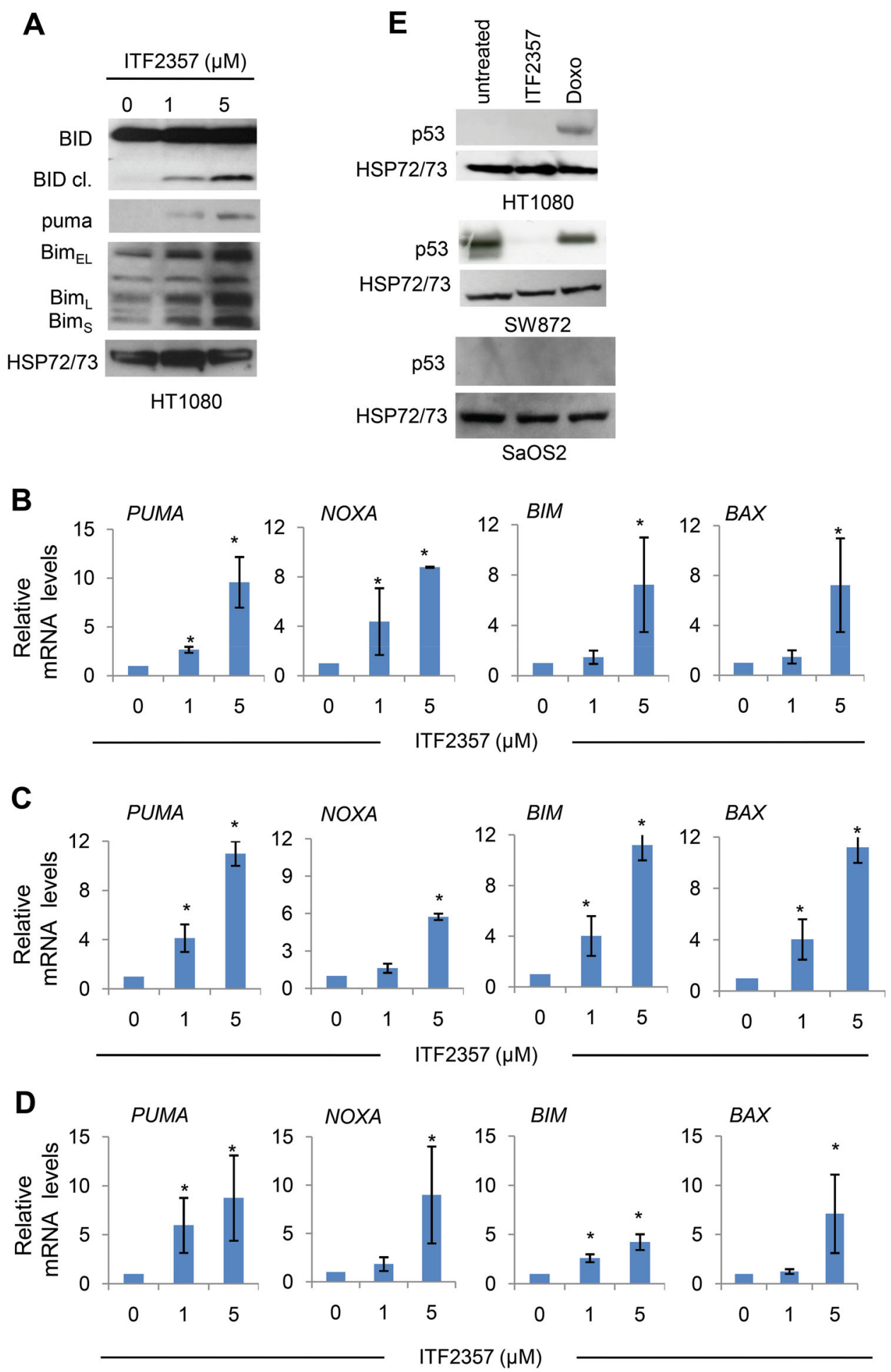

Fig. 2 ITF2357 activates mitochondrial apoptosis. a Western blot analysis of the indicated BH3-only pro-apoptotic proteins in total cell lysates from the HT1080 cell line treated with 1 and 5 MM ITF2357 for $48 \mathrm{~h}$. b-d Analysis of the indicated mRNA expression evaluated by qRT-PCR in HT1080 b, SW872 c and SaOS2 d cell lines exposed to 1 and $5 \mu$ M ITF2357 for $48 \mathrm{~h}$. Results are presented as the mean \pm SEM of at least two independent experiments. $p$ values were calculated between untreated and treated cells, ${ }^{*} p<0.05$. e Western blot analysis of p53 protein expression in total cell lysates from the indicated cell lines treated for $48 \mathrm{~h}$ with ITF2357 (1 $\mu \mathrm{M})$. As positive control of p53 induction, cells were exposed to Doxorubicin (Doxo, $0.1 \mu \mathrm{M}, 24 \mathrm{~h}$ ). a, e HSP72/73 expression was used as loading and transferring control. Western blots representative of two independent experiments with similar results are shown 


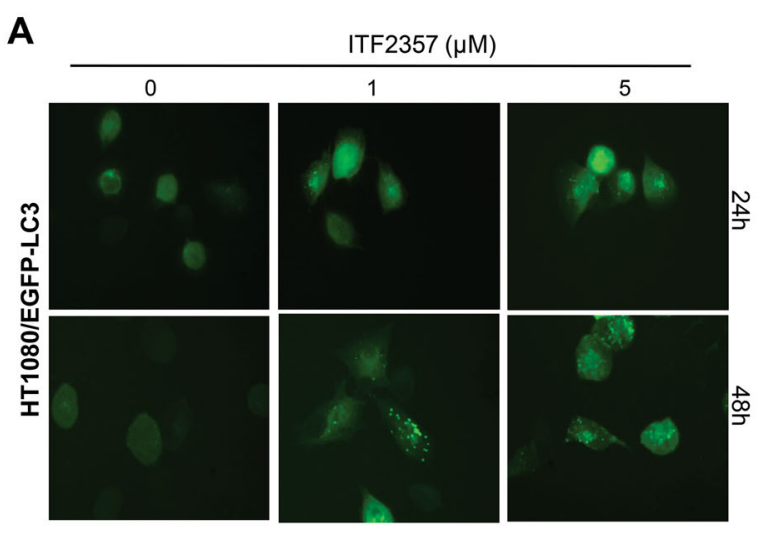

B
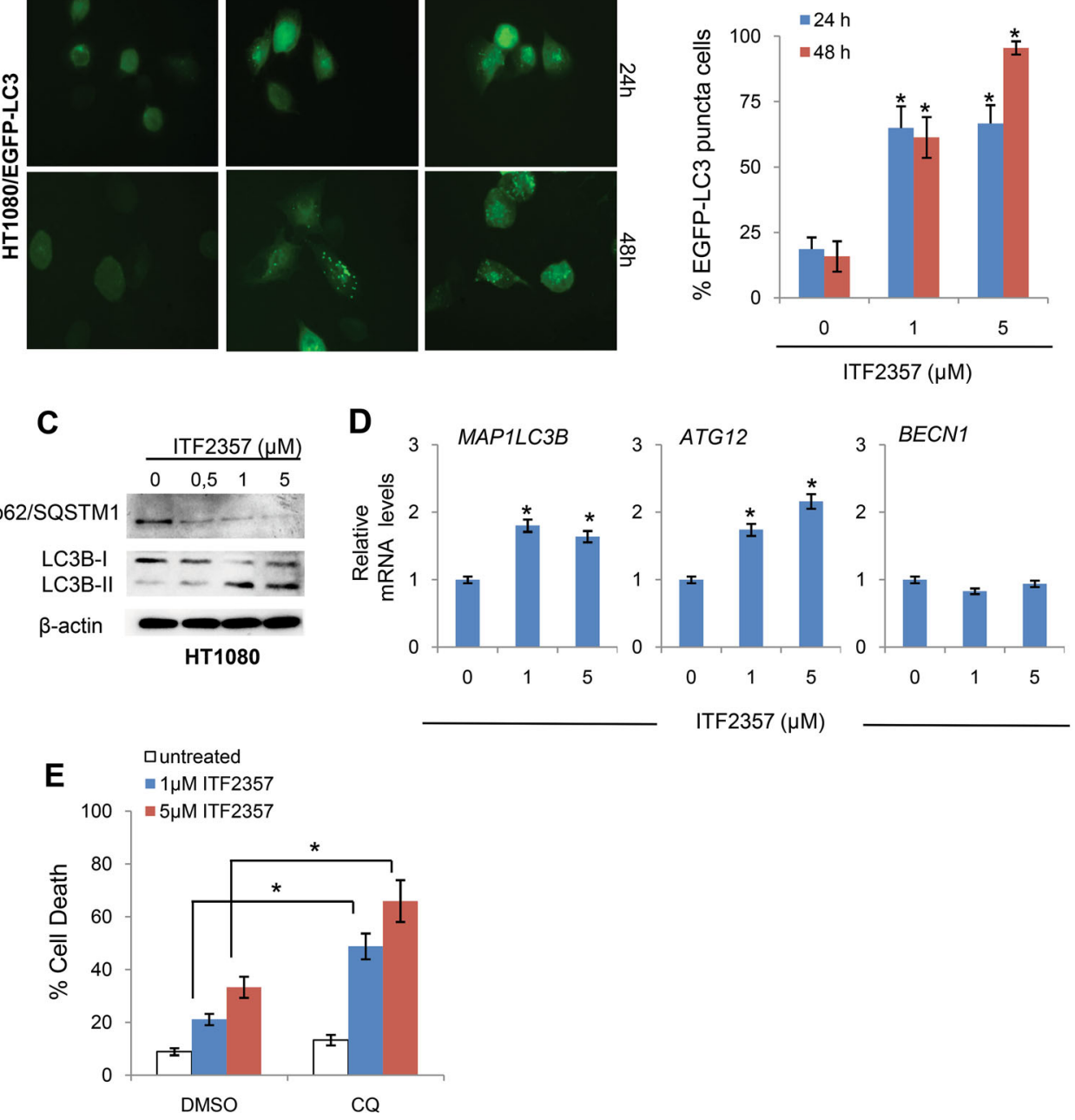

Fig. 3 ITF2357 induces a canonical autophagic process. a Representative images of autophagosomal structures by fluorescence microscopy in HT1080 cells stably transfected with EGFP-LC3B vector (HT1080/EGFP-LC3) and treated with 1 and $5 \mu$ M ITF2357 for the indicated times. b Quantification of cells positive for punctate autophagosomal structures treated as indicated in $\mathbf{a}$. The results represent the mean \pm SEM of three independent experiments. c Western blot analysis of microtubule-associated protein 1 (LC3B-I/II) conversion and of p62/SQSTM1 protein expression in total cell lysates from HT1080 cell line treated with increasing concentration of ITF2357 for $24 \mathrm{~h}$. Western blots representative of two independent experiments with similar results are shown. $\mathbf{d}$ Analysis of the indicated mRNA expression evaluated by qRT-PCR in HT1080 exposed to ITF2357 (1 and $5 \mu \mathrm{M}$ for $24 \mathrm{~h}$ ). Results are presented as the mean \pm SEM of at least two independent experiments. $p$ values were calculated between untreated and treated cells, $\left({ }^{*} p<0.05\right)$. e Analysis of cell death evaluated by PI staining in HT1080 cells exposed to ITF2357 for $48 \mathrm{~h}$ in absence or presence of Chloroquine (CQ), a late stage autophagy inhibitor, ${ }^{*} p<0.05$. The results represent the mean \pm SD of three independent experiments

anti-apoptotic protein bcl-2. Interestingly, overexpression of bcl-2 counteracted ITF2357-induced cell death, as assessed by cell permeabilization (Fig. 2b suppl.) and AnnexinV surface expression (Fig. 2c suppl.).

Next, we explored in HT1080 cells the expression levels of pro-apoptotic members of the bcl-2 family via western blot (Fig. 2a) and quantitative real-time PCR (qRT-PCR) (Fig. 2b) analyses. Treatment with ITF2357 resulted in a dose-dependent upregulation of Bim isoforms ( $\mathrm{Bim}_{\mathrm{EL}}$, $\mathrm{Bim}_{\mathrm{L}}$, and $\mathrm{Bim}_{\mathrm{S}}$ ). In addition, ITF2357 induced Noxa, Bax, and Puma expression and triggered the cleavage of BH3only protein Bid (tBid), suggesting tBid recruitment at mitochondria and its involvement in ITF2357-mediated apoptosis. Notably, ITF2357 also transcriptionally upregulated pro-apoptotic BH3-only proteins in SW872 cell line, harboring p53 mutation (Fig. 2c). Of note, we found that ITF2357 transcriptionally upregulated in a dosedependent manner pro-apoptotic BH3-only genes also in p53 null SaOS2 cells (Fig. 2d), indicating that in our experimental models, transcription of the pro-apoptotic 
BH3-only genes could involve factor(s) different from p53. Accordingly, ITF2357 induced a decrease of mut-p53 protein levels in SW872 cells, while no accumulation of p53 was observed after ITF2357 treatment of either HT1080 cells, bearing wt-p53, or p53 null SaOS2 cells (Fig. 2e).

Overall, these data indicate that ITF2357 induces apoptosis in a caspase- and bcl-2-dependent manner, through a p53-independent transcriptional mechanism.

\section{ITF2357 induces autophagy in human sarcoma cells}

Because it has been shown that ITF2357 induces autophagy in other cancer models ${ }^{36,37}$, we investigated the effect of ITF2357 on autophagy also in sarcoma cell lines. In HT1080 cells, the induction of autophagy by ITF2357 was confirmed based on the increase of autophagosomes formation, maturation, and degradation, as well as the increase of lipidated form of LC3B (LC3B-II) and the degradation of $\mathrm{p} 62 / \mathrm{SQTSM} 1$, indicating that the autophagic process is progressing to completion. In particular, we evaluated autophagy induction by analyzing LC3B redistribution in HT1080 cells stably expressing the EGFP-LC3B fusion protein (HT1080/EGFP-LC3). As shown in Fig. 3a, b, a time- and dose-dependent enhancement of punctate vesicular structures was observed in ITF2357-treated cells when compared to
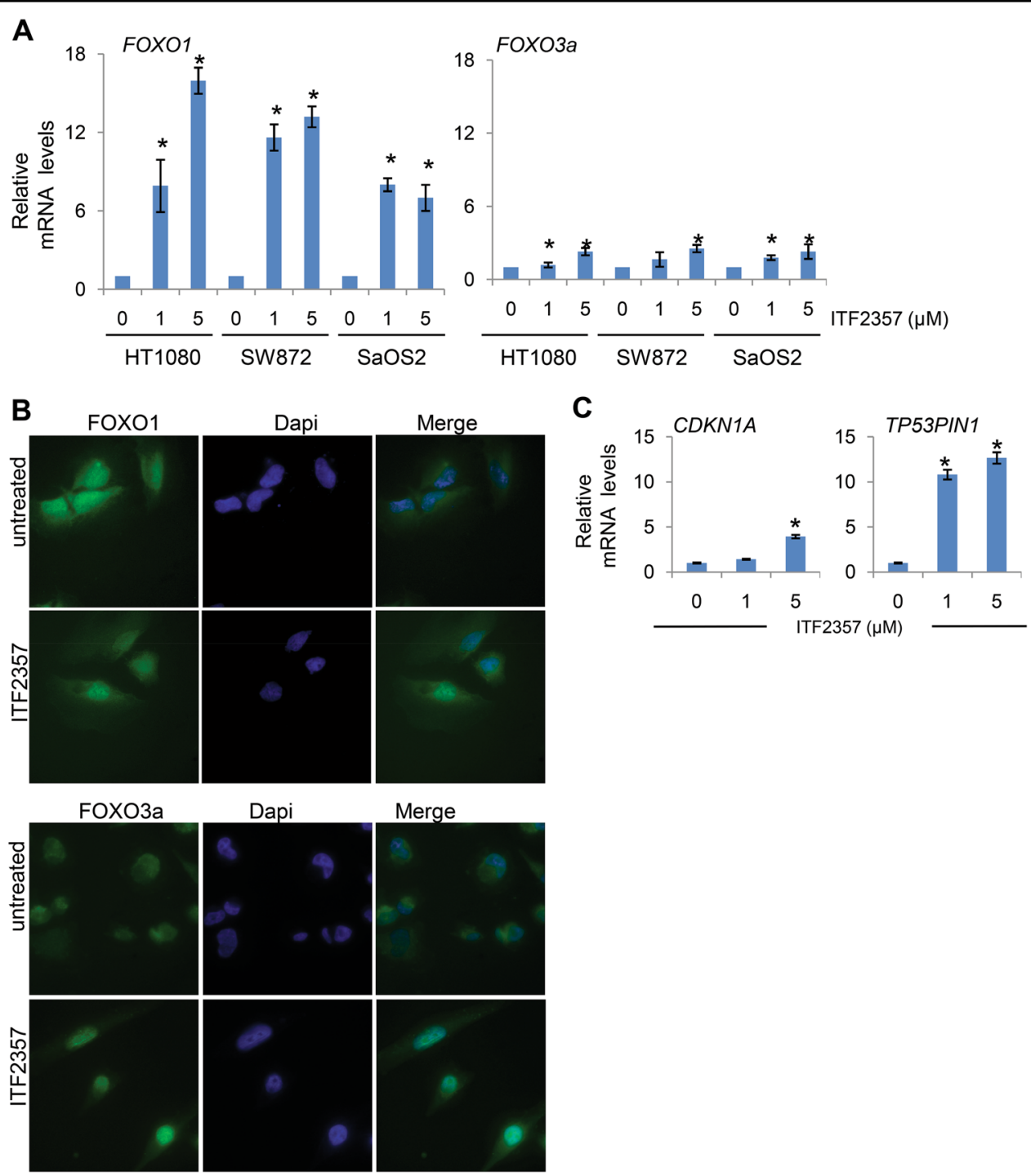

Fig. 4 ITF2357 induces FOXOs protein expression and transcriptional activity. a Analysis of FOXO1 and FOXO3 mRNA expression evaluated by qRT-PCR in the indicated cell lines treated for $24 \mathrm{~h}$ with 1 and $5 \mu \mathrm{M}$ ITF2357. b Representative images of immunofluorescence for FOXO1 and FOXO3 proteins in HT1080 cells after treatment with $1 \mu \mathrm{M}$ ITF2357. c qRT-PCR analysis of the indicated mRNA expression in HT1080 cells treated for $24 \mathrm{~h}$ with 1 and $5 \mu \mathrm{M}$ ITF2357. a, c Results are presented as the mean \pm SEM of two independent experiments. $p$ values were calculated between untreated and treated cells, ${ }^{*} p<0.05$ 
untreated ones. To further assess the autophagic flux in ITF2357-treated cells, we evaluated the autophagosome maturation in HT1080 cells expressing mRFP-EGFP-LC3 reporter (HT1080/ptf-LC3). Notably, ITF2357 treatment induced the autophagosome-lysosome fusion and complete autophagosome maturation (Fig. 2d suppl.). According with these results, we also found by western blot analysis an increase of LC3B-II form and a decrease of p62/SQTSM1 protein level upon treatment with ITF2357 (Fig. 3c).

Next upon ITF2357 treatment, we evaluated the transcriptional activation of $A T G 12, B E C N 1$, and MAP1LC3B genes, reported to be induced during the authophagic process $^{38}$. Interestingly, only ATG12 and MAP1LC3B messenger RNA (mRNA) were substantially upregulated in ITF2357-treated cells, while the expression level of

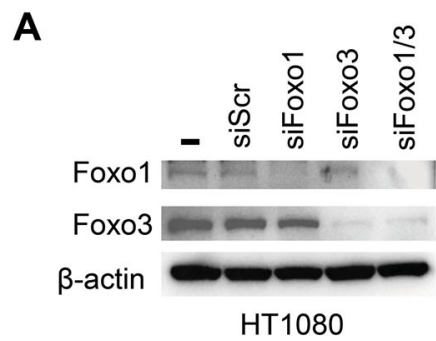

B
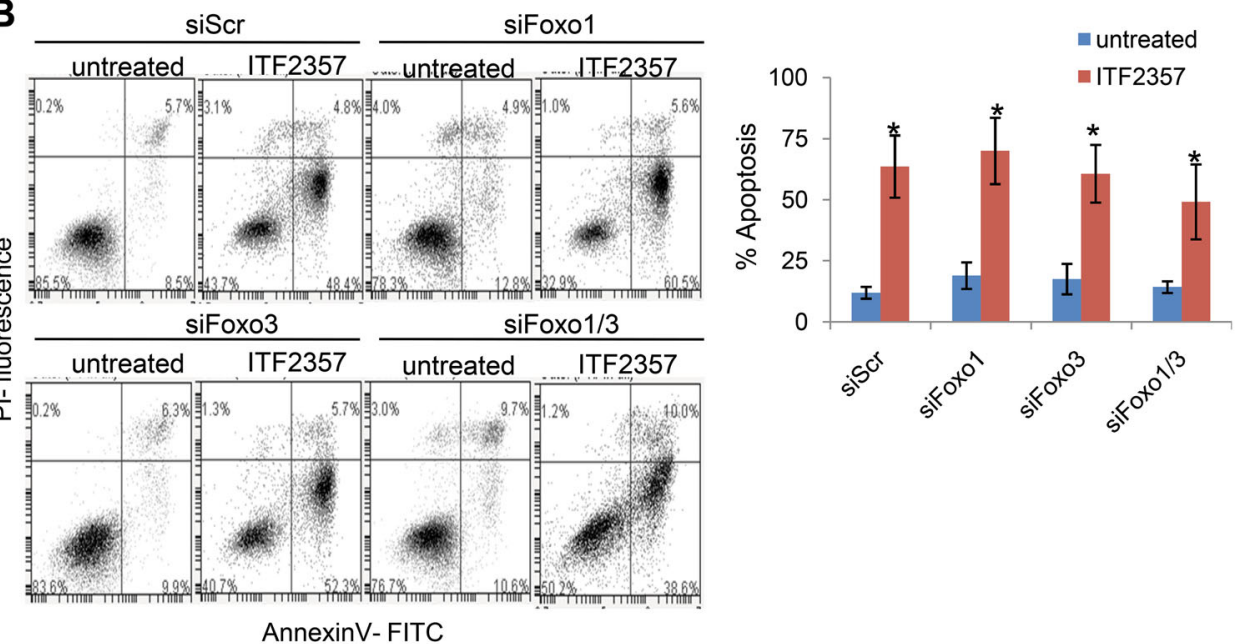

C

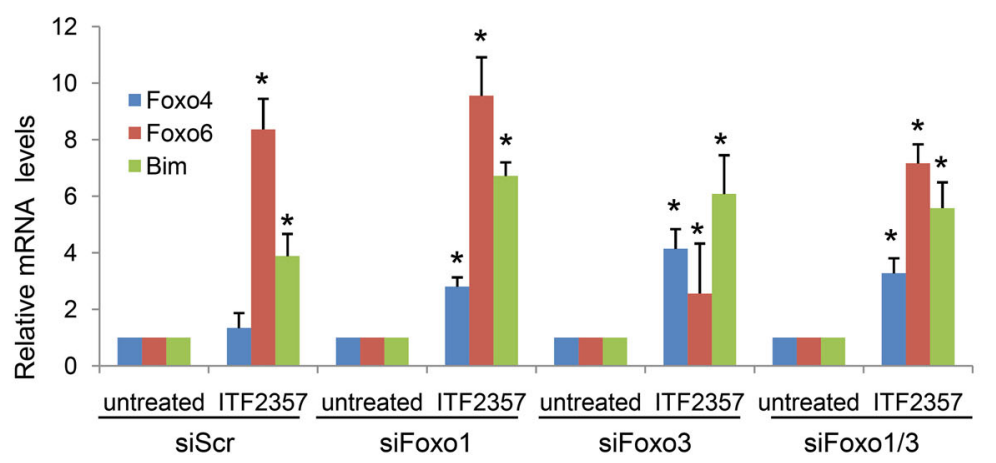

Fig. 5 Critical role of FOXOs protein in ITF2357-induced apoptosis. a Western blot analysis of FOXO1 and FOXO 3a protein expression in total cell lysates from HT1080 cells after $48 \mathrm{~h}$ from trasfection with siRNA-mediated knockdown of FOXO1 and FOXO3a, alone (siFOXO1 and siFOXO3) and in combination (siFOXO1/3) or with a control sequence (siScr). $\beta$-actin expression was used as loading and transferring control. Western blots representative of two independent experiments with similar results are shown. b Flow cytometric analysis (left panel) and quantification (right panel) of apoptotic cells by AnnexinV/PI staining HT1080 transfected with the indicated siRNAs (siScr, siFOXO1, siFOXO3a, and siFOXO1/3) and exposed to 1 $\mu M$ ITF2357 for $48 \mathrm{~h}$. The results represent the mean \pm SD of three independent experiments. c qRT-PCR analysis of the indicated mRNA expression in HT1080 transfected with the indicated siRNAs (siScr, siFOXO1, siFOXO3a, and siFOXO1/3) and exposed to $1 \mu \mathrm{M}$ ITF2357 for $48 \mathrm{~h} . p$ values were calculated between untreated and treated cells, ${ }^{*} p<0.05$ 
BECN1 mRNA was superimposable in untreated and treated cells (Fig. 3d). We also evaluated the biological outcome of autophagy inhibition upon ITF2357 treatment by using chloroquine (CQ), an inhibitor of the lysosomal $\mathrm{pH}$ gradient that blocks autophagy at late stage. As shown in Fig. 3e, quantification of cell viability showed an increased cell death when ITF2357 was combined with CQ. Similarly, ITF2357 treatment induced autophagy also in SW872 cells harboring mut-p53, as evidenced by analysis of LC3 puncta formation in SW872/EGFP-LC3B cells (Fig. 2e suppl.). These results indicate that ITF2357 induces a canonical autophagic process both in wt- and mut-p53 sarcoma cells and that ITF2357-induced autophagy shows a cell survival mechanism.

\section{Activation of Forkhead box (FOXOs) transcription factors has a critical role in ITF2357-induced cell death}

Several reports suggested a role for FOXO1 and FOXO3a proteins in regulating apoptosis through the expression of BH3-only target genes ${ }^{39}$. Thus, we tested FOXOs expression in three representative sarcoma cell lines exposed to ITF2357. As shown in Fig. 4a, FOXO1 and FOXO3a mRNA levels were increased in three different sarcoma cell lines treated with ITF2357. Next, we analyzed FOXOs nuclear and cytosolic localization in HT1080 cells upon ITF2357 exposure. As reported by immunofluorescence analysis, a similar localization of FOXO1 and FOXO3a was observed in HT1080 cells after ITF2357 treatment (Fig. 4b). In particular, ITF2357treated cells showed a more evident nuclear localization of FOXO3a in comparison to untreated ones. To further confirm the transcriptional activity of FOXOs, we analyzed the transcription of CDKN1A/p21 and TP53PIN1, two other well-known FOXO-target genes. As reported in Fig. 4c, an increased expression of the two transcripts was observed after ITF2357 treatment. These results suggest that FOXOs might play a role in ITF2357-induced regulation of apoptosis. Thus, we proceeded to confirm the relevance of FOXOs in ITF2357-mediated apoptosis by testing the consequences of siRNA-mediated knockdown of FOXO1 and FOXO3a, alone and in combination (Fig. 5a). Surprisingly, knockdown of FOXO1 or FOXO3a is not associated with a decrease of apoptotic rates in HT1080 cells treated with ITF2357. Notably, similar results were obtained in HT1080 cells after simultaneous knockdown of both FOXO1 and 3a (Fig. 5b). These results were also corroborated by analysis of Bim expression in HT1080 cells upon FOXOs silencing and ITF2357 treatment. Indeed, after FOXOs silencing, Bim expression was still upregulated in response to ITF2357 (Fig. 5c). Interestingly, we found that mRNA of other two FOXO members, FOXO4 and FOXO6, were upregulated in response to ITF2357 when FOXO1 and 3a were downregulated. These results suggest that upregulation of
FOXO4 and 6 proteins might sustain ITF2357-mediated apoptosis by upregulation of pro-apoptotic BH3-only protein Bim (Fig. 5c).

\section{ITF2357 sensitizes human sarcoma cells to doxorubicin treatment both in vitro and in vivo}

Based on these data, we decided to explore the effect of ITF2357 in combination with conventional sarcoma chemotherapy, such as Doxo, a topoisomerase II inhibitor frequently used as first-line chemotherapeutic for sarcomas $^{3}$. Sarcoma cell lines were exposed to ITF2357 or Doxo alone or in combination (ratio 5:1) and their effect on cell viability was evaluated. Next, combination index (CI) was also calculated by conservative isobologram analysis to evaluate the pharmacological interaction between ITF2357 and Doxo. As shown by growth inhibition curves (Fig. 6a), simultaneous treatment with ITF2357 and Doxo resulted in a higher effect when compared to single treatments, in all cell lines analyzed, although to a different extent. In particular, in HT1080 cells, the combination of ITF2357 $(0.5 \mu \mathrm{M})$ and Doxo $(0.1$ $\mu \mathrm{M})$ caused a growth reduction of about $90 \% \pm 4.7$, while single treatments resulted in $50 \% \pm 3$ and $63 \% \pm 10$ growth reduction after treatment with ITF2357 or Doxo, respectively. A similar effect was also observed in SW872 and SW982 cells. As reported in Table 1, ITF2357 acted in a synergistic manner with Doxo in seven different cell lines. Notably, a significant growth-inhibitory effect of ITF2357 in vitro and a synergistic interaction with Doxo was also evidenced in two-patient-derived sarcoma cell lines (LSP1, 4052), while the effect of the two drugs was additive in 3844B patient-derived sarcoma cell line (Table 1, Fig. 6b). We analyzed modulation of apoptosis following single or combined treatment of two representative cell lines, HT1080 and SW982. In agreement with cell viability data, Annexin V staining shows that all the assessed cell lines have the highest amount of early apoptotic cells (Annexin V+/PI- cells) after combined treatment when compared to the corresponding single treatments (Fig. 6c).

The in vitro synergistic cytotoxic activities of Doxo and ITF2357 observed in multiple sarcoma cell lines suggested a therapeutic potential in vivo. To test this hypothesis, we treated established SW982 soft tissue sarcoma ${ }^{40}$ xenografts for 4 weeks with vehicle, Doxo $(2.2 \mathrm{mg} / \mathrm{kg}$ per week), ITF2357 (100 mg/kg per day for 4 days), or combination Doxo/ITF2357 (weekly Doxo followed by daily ITF2357 for 4 days). As reported in Fig. 7a, treatment with either Doxo or ITF2357 alone did not significantly affect xenografts growth. On the contrary, the combined treatment was significantly inhibitory and was highly tolerated, as no significant weight loss, diet consumption, and postural and behavioral changes were observed. In vivo experiments were also performed in HT1080 xenografts 

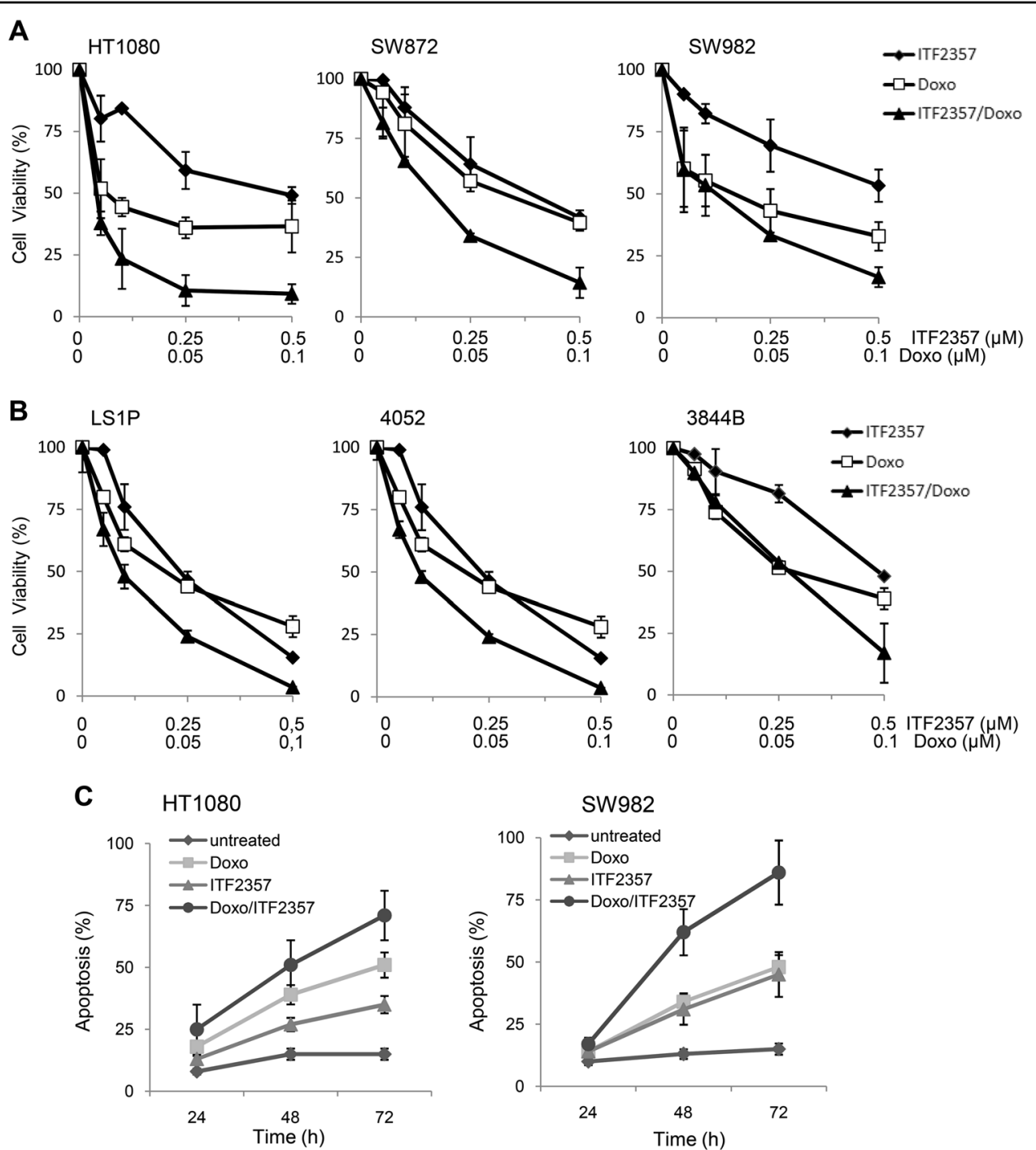

Fig. 6 ITF2357 sensitizes human sarcoma cells to Doxorubicin treatment. a, b Analysis of cell viability by MTT assay in the indicated established $\mathbf{a}$ and patient-derived $\mathbf{b}$ cell lines treated with ITF2357 and Doxorubicin (Doxo, drug ratio 5:1) alone or in combination. The results are reported as "viability of drug-treated cells/viability of control cells" $\times 100$ and represent the mean \pm SD of three independent experiments performed in triplicate. c Cytofluorimetric analysis of apoptotic cells in the indicated cell lines treated with ITF2357 (0.5 $\mu \mathrm{M})$ and Doxo $(0.1 \mu \mathrm{M})$ alone or in combination for 24 , 48 , and $72 \mathrm{~h}$. The results are reported as mean \pm SD of three independent experiments

in which treatment with either Doxo or ITF2357 alone slightly affects xenografts growth (about 15\% of inhibition). On the contrary, the combined treatment reduces HT1080 tumor growth of about 30\% when compared to control xenografts (data not shown). Accordingly, with in vitro data, immunohistochemical analysis of SW982 tumor xenografts showed an increased percentage of apoptotic cells in combination treatments when compared to single ones (Fig. $7 \mathrm{~b}, \mathrm{c}$ ). In particular, TUNELpositive staining nuclei revealed an apoptotic index of $5 \%$ for control, 13.6\% for Doxo, 13.4\% for ITF2357, and 23.6\% for the combination. These results indicate that ITF2357 sensitizes sarcoma from different histologic subtypes and p53 mutational status to Doxo both in vitro and in vivo.

\section{Discussion}

Novel therapies are urgent to improve the treatment of sarcomas, a group of different tumors that show a poor prognosis and a low curative outcome ${ }^{1,4}$.

Using in vitro and in vivo sarcoma models, here we demonstrate that the HDACi, ITF2357, decreases cell viability, activates apoptosis, and increases the growthinhibitory efficacy of Doxo. We also show evidence about the molecular mechanisms of action of ITF2357-mediated cell death, which implied the activation of mitochondrial 
pathway of apoptosis, as attested by the increase of proapoptotic BH3-only proteins, and both caspase- and bcl2-dependent cell death. BH3-only proteins are effectors of mitochondrial apoptosis and are able to activate Bax and Bak through direct or indirect mechanisms ${ }^{41}$. In agreement with other groups demonstrating p53-independent transcriptional regulation of some pro-apoptotic BH3only genes ${ }^{42}$, we found that ITF2357 transcriptionally upregulated, in a dose-dependent manner, Bax, Puma, Noxa, and Bim in cells harboring mutant or wt p53, thus indicating a p53-independent mechanism.

Table1 Response to ITF2357 and Doxorubicin in combination of human sarcoma cells lines

\begin{tabular}{lllll}
\hline & & \multicolumn{3}{l}{ Cl values at } \\
\cline { 3 - 5 } Cell line & Subtype & ED50 & ED 75 & ED90 \\
\hline HT1080 & Fibrosarcoma & 0.40 & 0.04 & 0.02 \\
SW982 & Synovial sarcoma & 0.86 & 0.43 & 0.30 \\
SW872 & Liposarcoma & 0.76 & 0.85 & 0.96 \\
SaOS2 & Osteosarcoma & 0.73 & 0.85 & 1.04 \\
U2OS & Osteosarcoma & 0.64 & 0.62 & 1.02 \\
SKLMS & Leiomyosarcoma & 0.16 & 0.09 & 0.08 \\
A204 & Rabdomyosarcoma & 0.52 & 0.51 & 0.98 \\
3844b & Osteosarcoma & 1.08 & 1.00 & 0.95 \\
4052 & Condrosarcoma & 0.98 & 0.80 & 0.76 \\
LS1P & Liposarcoma & 0.86 & 0.83 & 0.84 \\
\hline
\end{tabular}

$C l$ combination index, ED effective dose
The notion that activation of mitochondrial pathway occurs upstream and before caspases activation is corroborated by results obtained in Bcl- 2 overexpressing cells, and by the use of zVAD, a broad-range caspase inhibitor: both bcl-2 overexpression and caspases inhibition strongly reduced apoptosis. Interestingly, we also found that ITF2357 induces a canonical autophagic process, and that inhibition of autophagy strongly increased apoptosis induced by ITF2357, suggesting that in our models, autophagy shows a pro-survival effect. Controversial results have been reported about the effect of autophagy on HDACi-mediated cell death, being observed both cytoprotective and cytotoxic functions of autophagy ${ }^{43,44}$. We previously reported that ITF2357 in combination with Pemetrexed caused a toxic form of autophagy that subsequently activated a caspase-dependent apoptotic program in lung cancer models ${ }^{37}$. In agreement with other studies, we found that autophagy shows a prosurvival effect. Among them, a recent study shows that FOXO1dependent pathways are implicated in HDACi-induced autophagy $^{26}$. FOXOs members, include transcription factors with relevant role in gene regulation ${ }^{39}$. They act as tumor suppressor genes and their alterations have been reported in some human cancers ${ }^{39}$, including rhabdomyosarcoma and osteosarcoma ${ }^{45,46}$. We reported that HDAC inhibition by ITF2357, induced FOXO1/3 upregulation, FOXO proteins nuclear accumulation, and transcriptional activation of FOXO-target genes, such as BH3-only proteins Puma, Noxa, and Bim, thus altering the equlibrium between anti-apoptotic and pro-apoptotic proteins belonging to bcl-2 family, which in turn culminates into mitochondrial alteration, caspases activation, and DNA fragmentation. These data are in agreement
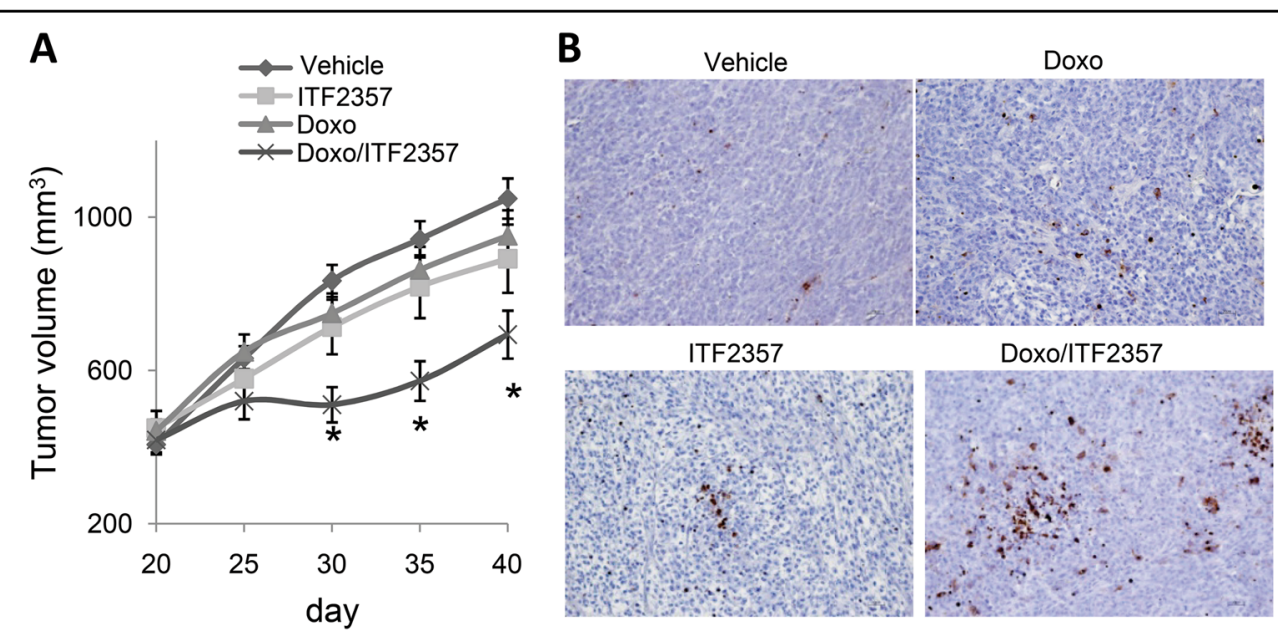

Fig. 7 ITF2357 reduces tumor growth and potentiates Doxorubicin effect in vivo. a In vivo response of SW982 xenograft to vehicle (daily), Doxorubicin (Doxo, $2.2 \mathrm{mg} / \mathrm{kg}$ per week) or ITF2357 (100 mg/kg per day for 4 days) alone, or in combination (weekly Doxo followed by daily ITF2357 for 4 days). Treatments lasted 4 weeks. $\mathbf{b}$ Representative images of immunohistochemical detection of apoptosis by TUNEL assay of tumor xenografts treated as reported in a 
with the well-established pro-apoptotic function of FOXO1, through upregulation of Bim or Noxa genes recently described in osteosarcoma ${ }^{46}$. Also the expression of other downstream targets of FOXOs, including CDKN1A/p21 and TP53PIN1, was found to be upregulated in response to ITF2357 in sarcoma models. Knockdown experiments were performed to deeper investigate the role of FOXOs in cytotoxicity mediated by ITF2357. Notably, reduction of both FOXO1 and 3a did not inhibit the induction of Bim expression in response to ITF2357, and consequently did not protect sarcoma cells against ITF2357-induced apoptosis. Since we also observed that in silenced cells, ITF2357 treatment upregulated other two members of FOXO family, FOXO4 and FOXO6, we might hypothesize that FOXO4 and FOXO6 upregulation might sustains ITF2357-mediated apoptosis through upregulation of pro-apoptotic $\mathrm{BH} 3$-only protein Bim. Overall, our results evidence similar FOXOdependent apoptotic mechanisms in response to ITF2357 in sarcoma cells with wt or mutated p53 status.

We also studied the role of ITF2357 in combinatorial regimes with Doxo, the current standard of systemic therapy care for most subtypes of sarcoma. Overall our results demonstrate that (1) co-treatment ITF2357/Doxo strongly inhibits cell viability and determines a significant increase in number of apoptotic cells when compared to single treatments; (2) as evidenced by $\mathrm{CI}$ analysis, there is a synergistic interaction of ITF2357 and Doxo to reduce cell viability and induce apoptosis; (3) co-treatment ITF2357/Doxo strongly reduces in vivo tumor growth, whereas monotherapy with either agents exhibit no significant anti-tumor effect; and (4) the clinical relevance of co-treatment ITF2357/Doxo is also evidenced in primary patient-derived sarcoma cells. These evidences demonstrate the potency of this combination therapy in sarcoma models and are in agreement with other sarcoma stu$\operatorname{dies}^{9,29,47 .}$.

Taken together, our data indicate that ITF2357 may represent an important potential therapeutic agent against human sarcoma regardless of p53 status, and that the pharmacological combination of ITF2357 with Doxo has the potential to enhance sensitization in different preclinical models of sarcoma. Based on these results, the potential clinical value of ITF2357, over other HDACi agents previously assessed in sarcoma, deserves further studies in the future.

\section{Materials and methods}

\section{Cell culture and chemicals}

Established osteosarcoma (SaOS2, U2OS), liposarcoma (SW872), synovial sarcoma (SW982), rabdomyosarcoma (A204), leiomyosarcoma (SKLMS), and fibrosarcoma (HT1080) cell lines were originally purchased from ATCC (Manassas, VA). Patient-derived osteosarcoma (3844B), liposarcoma (LS1P), and condrosarcoma (4052) cells were obtained from Dr Baldini (Istituto Ortopedico Rizzoli, Bologna, Italy). EGFP-LC3B, ptf-LC3, and GFP-bcl-2 stable clones, obtained as previously described ${ }^{48}$, were cultured in the presence of geneticin $(800 \mu \mathrm{g} / \mathrm{ml}$, SigmaAldrich, St. Louis, MO). Cells were routinely tested for mycoplasm presence.

ITF2357 (diethyl-[6-(4-hydroxycarbamoyl-phenyl carbamoyloxymethyl)-naphthalen-2-yl methyl]-ammonium chloride monohydrate) was provided by Italfarmaco (patent WO 97/43251, US 6034096, Milan, Italy). Doxorubicin (Doxo) was obtained from Sigma-Aldrich. Pancaspase inhibitor zVAD-fmk (zVAD, $50 \mu \mathrm{M}$, SigmaAldrich) and chloroquine diphosphate (CQ, $25 \mu \mathrm{M}$, Sigma-Aldrich) were dissolved in DMSO and water, respectively.

\section{Analysis of cell viability and apoptosis}

The effect of ITF2357 and Doxo on in vitro cell proliferation was assessed following manufacturer's protocol by measuring 3-[4,5-dimethylthiazol-2-yl]-2,5-diphenyltetrazolium bromide inner salt (MTT, Sigma-Aldrich) dye absorbance. The viability was calculated for each concentration of drugs used as "optical density (OD) of treated cells/OD of control cells $\times 100$." By using Calcusyn software, we calculated the concentration of drug that reduces $50 \%$ of cell viability (IC50). According to pilot experiments, the DMSO concentration $(0.05 \%)$ used for the experiments did not affect the proliferation of sarcoma cell lines (data not shown). Caspase-3 activation and the presence of apoptotic cells were evaluated in untreated or treated cells staining using active caspase 3PE antibody (cat. 559565, BD Bioscience, San Jose, CA) and with Annexin V-FITC or AnnexinV-APC and PI staining. Cytofluorimetric analysis was performed by BD C6 Accuri as previously reported ${ }^{37}$.

\section{Analysis of autophagy}

LC3B puncta formation (autophagosomal structures) in EGFP-LC3B-expressing cells, and distribuition/alteration of fluorescent signals (autophagosome maturation) in mRFP-GFP-LC3B-expressing cells were detected by fluorescence microscopy after grown of cells on glass coverslips and fixation formaldehyde $(2 \%, 10 \mathrm{~min}$, room temperature $)^{38}$.

\section{Western blot and immunofluorescence analyses}

Western blot and immunofluorescence analyses were performed as previously described ${ }^{37,48}$ using the following antibodies: $\mathrm{H} 3$ acetylated histone (cat. 06-599), FOXO3 (cat. 04-1007) (Millipore, Billerica, MA), p62/ SQSTM1 (cat. sc-28369), p53 (cat. sc-126), Bax (cat. sc493), Noxa (cat. sc-30209) (Santa Cruz, Biotechnology, Santa Cruz, CA), HSP72/73 (cat. Hsp-01, Calbiochem, 
San Diego, CA), acetyl- $\alpha$-tubulin (cat. T7451), Puma (cat. PRS3041)(Sigma-Aldrich), LC3B (cat. 2775S), FOXO1 (cat. 2880) and BID (cat. 20025), Bim (cat. 2819) (Cell Signaling, Danvers, MA). Goat anti-mouse IgG and goat anti-rabbit IgG conjugated to horseradish peroxidase were used as secondary antibodies. Enhanced chemiluminescence was used for detection (Amersham Bioscience, Freiburg, Germany). Representative blots of at least two independent experiments are shown.

\section{Assessment of synergism between ITF2357 and Doxo}

Effects of drugs combination were evaluated using the CI equation through CalcuSyn software (Biosoft, Cambridge, UK). Synergism $\mathrm{CI}<0.9$, additivity $0.9<\mathrm{CI}<1.1$, antagonism $\mathrm{CI}>1.1$.

\section{Quantitative RT-PCR analysis}

Quantitative real-time PCR analysis was performed to evaluate gene expression, as previously reported ${ }^{37}$. The following primers were used: human Puma forward: $5^{\prime}$ AAGTCAGGACTTGCAGGCGCG-3'; human Puma reverse: 5'-TGGGTCCCAGTCCGTGTGTGT-3'; human Bax forward: 5'-TCCCGGCTCTCTGATCCCCG-3'; human Bax reverse: 5'-GGCTAGGGGAACGCTATATGC-3'; human Noxa forward: 5'-CGCTGACGACGTCCCAGCGTTT-3'; human Noxa reverse: 5'CGAAGACGGCGTTAT-3'; human Bim forward: $5^{\prime}$ CAGAGATATGGATCGCCCAAG-3'; human Bim reverse: $\quad 5^{\prime}$-TGCGTCTGCTACAGTGGTTCTT-3'; human MAP1LC3B forward: $5^{\prime}$ TGGCCCAACCTGTCTGTACTTC-3'; human MAP1LC3B reverse: 5'-AAAAGTACCAGCCGCATGGAG-3'; human ATG12 forward: 5'-GCAGAAAAGGTTAGGCGTTTTG-3'; human ATG12 reverse: 5'-ATGTGCTTGCTCTCCTGGCTA-3'; human BECN1 forward: $5^{\prime}$-AGGAACTCACAGCTCCATTAC3'; human BECN1 reverse: 5'-AATGGCTCCTCTCCTGAGTT-3'; human FOXO1 forward: 5'-AACCTGGCATTACAGTTGGCC-3'; human FOXO1 reverse: $5^{\prime}$ AAATGCAGGAGGCATGACTACG-3'; human FOXO3a forward: $5^{\prime}$-TGCGTGCCCTACTTCAAGGAT3'; human FOXO3a reverse: 5'-ACCCGCATGAATCGACTATGC-3'; human TP53PIN1 forward: 5'GAGGTTGTCACCAACGCACGT-3'; human TP53PIN1 reverse: 5'-TGAGGGAGAGATCCACCTCTG-3'; human CDKN1A forward: 5'-CCTGGCACCTCACCTGCTCTGCTG-3'; human CDKN1A reverse: 5'GCAGAAGATGTAGAGCGGGCCTTTG-3'; human FOXO4 forward: 5'ACTGTGGCAGGCTTCACTGAAC3'; human FOXO4 reverse: 5'TCTAGGTCTATGATCGCGGCA-3'; human FOXO6 forward: 5'TCTACGACTGGATGGTCCGT-3'; human FOXO6 reverse: 5'GGGTCTTCCCTGTCTTTCCG-3'; human GAPDH forward: 5'-TCCCTGAGCTGAACGGGAAG-3'; human
GAPDH reverse: 5'-GGAGGAGTGGGTGTCGCTGT-3'; human actin: forward: 5'-ATTGCCGACAGGATGCAGAA-3'; human actin reverse: 5'- GCTGATCCACATCTGCTGGAA-3'.

Each sample was run in triplicate and normalized to GAPDH or actin mRNA to evaluate relative expression. The $2^{-\Delta \Delta \mathrm{Ct}}$ method was used to evaluate fold change in gene expression levels, expressed in unit less values.

\section{In vivo experiments}

Procedures relative to animal use and care were authorized and certified by Italian Minister of Health (decree no. 67/97A, protocol 2560/97, Rome Health Service Unit). Regina Elena National Cancer Institute and animal care Unit approved all the procedures involving animals (species, quality and number of animals, discomfort/distress/pain of animals, anesthesia and killing).

SW982 of $5 \times 10^{6}$ or HT1080 cells were intramuscularly injected into immunodeficient athymic mice (6-8 weekold female). Four groups of animals with similar tumor volume were created when the tumors reach the palpability. The following treatments administered for 4 weeks: (1) daily vehicle administration, (2) weekly intraperitoneal treatment with Doxo $(2.2 \mathrm{mg} / \mathrm{kg})$, (3) daily oral administration with ITF2357 (100 mg/kg) for 4 days, and (4) sequential Doxo/ITF2357 treatment (weekly Doxo followed by daily ITF2357 for 4 days). Ten animals composed each group. Tumor volume $\left(\mathrm{mm}^{3}\right)$ was daily calculated to evaluate the effect of different treatments on in vivo tumor growth. Animals were also daily monitored for food consumption, body weight, and behavior.

The experiments were repeated twice. At the end of treatment, tumors were excised, fixed in formalin, embedded in paraffin, and stained with hematoxylin and eosin. Tumor inhibition rate (\%) was calculated as follows: (mean tumor volume of control group-mean tumor volume of treated group)/mean tumor volume of control group $\times 100 \%$. The non-parametric Mann-Whitney test was employed to evaluate differences between the groups. Differences were statistically significant when $p<0.05$.

TUNEL assay (In Situ Cell Death Detection Kit, POD, Roche) was used to detect apoptosis in tumor sections according to the manufacturer's instructions. Frozen sections of $5 \mu \mathrm{m}$ ( 3 for each tumor) were analyzed by light microscopy and apoptotic cells were counted in four highpower fields ( $\times 400$ magnification).

\section{Statistical analysis}

All experiments were repeated at least two times. Cell viability, apoptosis, and mRNA levels were expressed as means \pm SD or means \pm SEM, and significances were analyzed with the $t$ test. All data were included in the analyses. Based on variation shown in our previous results, we determined the sample sizes by using power 
analysis ${ }^{37}$. Immunofluorescence and TUNEL analyses were performed by two investigators in blinded and independent manner.

\section{Acknowledgements}

We thank Dr. Adele Petricca for manuscript preparation. Supported by Grants from Italian Association for Cancer Research (DDB IG 18560, NB IG 11426) and IRE Translational Sarcoma Group (881/2015). M.G.T. is recipient of a fellowship from FIRC.

\section{Author details}

'Preclinical Models and New Therapeutic Agents Unit, IRCCS Regina Elena National Cancer Institute, Rome, Italy. ${ }^{2}$ Pathology Unit, IRCCS Regina Elena National Cancer Institute, Rome, Italy. ${ }^{3}$ Cellular Networks and Molecular Therapeutic Targets Unit, IRCCS Regina Elena National Cancer Institute, Rome, Italy. ${ }^{4}$ Medical Oncology, IRCCS Regina Elena National Cancer Institute, Rome, Italy. ${ }^{5}$ Orthopaedic Pathophysiology and Regenerative Medicine Unit, Istituto Ortopedico Rizzoli, Bologna, Italy. ${ }^{6}$ Department of Biomedical and Neuromotor Sciences, University of Bologna, Bologna, Italy. ${ }^{7}$ Oncological Orthopedics, IRCCS Regina Elena National Cancer Institute, Rome, Italy. Institute of Molecular Biology and Pathology, National Research Council, Rome, Italy

\section{Author contributions}

M.D.M., M.D., M.G.T., B.A., and C.M. performed the experiments; D.D.B., M.M., V.F., and R.B. edited the manuscript; R.F. and N.B. provided the cell lines; S.B., M.D.M. and M.D. analyzed the data; D.T. conceived, designed, performed the experiments, and wrote the manuscript; D.D.B. supervised the study and wrote and edited the manuscript; all authors read and approved the final manuscript.

\section{Conflict of interest}

The authors declare that they have no conflict of interest.

\section{Publisher's note}

Springer Nature remains neutral with regard to jurisdictional claims in published maps and institutional affiliations.

Supplementary Information accompanies this paper at (https://doi.org/ 10.1038/s41389-018-0026-x).

Received: 16 May 2017 Revised: 26 September 2017 Accepted: 9 November 2017

Published online: 23 February 2018

\section{References}

1. HaDuong, J. H., Martin, A. A., Skapek, S. X. \& Mascarenhas, L. Sarcomas. Pediatr. Clin. North Am. 62, 179-200 (2015).

2. Zahm, S. H. \& Fraumeni, J. F. Jr The epidemiology of soft tissue sarcoma. Semin. Oncol. 24, 504-514 (1997).

3. Walczak, B. E. \& Irwin, R. B. Sarcoma chemotherapy. J. Am. Acad. Orthop. Surg. 21, 480-491 (2013).

4. Riedel, R. F. Systemic therapy for advanced soft tissue sarcomas: highlighting novel therapies and treatment approaches. Cancer 118, 1474-1485 (2012).

5. Le Cesne, A. et al. Doxorubicin-based adjuvant chemotherapy in soft tissue sarcoma: pooled analysis of two STBSG-EORTC phase III clinical trials. Ann. Oncol. 25, 2425-2432 (2014)

6. Di Marcotullio, L., Canettieri, G., Infante, P., Greco, A. \& Gulino, A. Protected from the inside: endogenous histone deacetylase inhibitors and the road to cancer. Biochim. Biophys. Acta 1815, 241-252 (2011).

7. Lane, A. A. \& Chabner, B. A. Histone deacetylase inhibitors in cancer therapy. J. Clin. Oncol. 27, 5459-5468 (2009).

8. Tan, J., Cang, S., Ma, Y., Petrillo, R. L. \& Liu, D. Novel histone deacetylase inhibitors in clinical trials as anti-cancer agents. J. Hematol. Oncol. 3, 5 (2010).

9. Choy, E. et al. Phase 1 study of oral abexinostat, a histone deacetylase inhibitor, in combination with doxorubicin in patients with metastatic sarcoma. Cancer 121, 1223-1230 (2015)
10. Chu, Q. S. et al. A phase II study of SB939, a novel pan-histone deacetylase inhibitor, in patients with translocation-associated recurrent/metastatic sarcomas-NCIC-CTG IND 200t. Ann. Oncol. 26, 973-981 (2015).

11. Thomas, S. et al. A phase I trial of panobinostat and epirubicin in solid tumors with a dose expansion in patients with sarcoma. Ann. Oncol. 27, 947-952 (2016).

12. Kayarthodi, S. et al. Anti-epileptic drug targets ewing sarcoma. J. Pharm. Sci. Pharmacol. 1, 87-100 (2014).

13. Sampson V. B. et al. Vorinostat enhances cytotoxicity of SN-38 and temozolomide in ewing sarcoma cells and activates STAT3/AKT/MAPK pathways. PLoS ONE 10, e0142704 (2015)

14. Lopez, G. et al. HDAC inhibition for the treatment of epithelioid sarcoma: novel cross talk between epigenetic components. Mol. Cancer Res. 14, 35-43 (2016).

15. Ou, W. B. et al. HDACi inhibits liposarcoma via targeting of the MDM2-p53 signaling axis and PTEN, irrespective of p53 mutational status. Oncotarget $\mathbf{6}$, 10510-10520 (2015).

16. Ma, L. et al. SIRT1 and SIRT2 inhibition impairs pediatric soft tissue sarcoma growth. Cell Death Dis. 5, e1483 (2014).

17. Laporte, A. N. et al. HDAC and proteasome inhibitors synergize to activate proapoptotic factors in synovial sarcoma. PLoS ONE 12, e0169407 (2017).

18. Su, L., Cheng, H., Sampaio, A. V., Nielsen, T. O. \& Underhill, T. M. EGR1 reactivation by histone deacetylase inhibitors promotes synovial sarcoma cell death through the PTEN tumor suppressor. Oncogene 29, 4352-4361 (2010).

19. Capobianco, E. et al. Separate and combined effects of DNMT and HDAC inhibitors in treating human multi-drug resistant osteosarcoma HosDXR150 cell line. PLoS ONE 9, e95596 (2014).

20. Murahari, S. et al Sensitivity of osteosarcoma cells to HDAC inhibitor AR-42 mediated apoptosis. BMC Cancer 17, 67 (2017).

21. Pettke, A. et al. Suberanilohydroxamic acid (vorinostat) synergistically enhances the cytotoxicity of doxorubicin and cisplatin in osteosarcoma cell lines. Anticancer Drugs 27, 1001-1010 (2016).

22. Thayanithy, $V$. et al. Combinatorial treatment of DNA and chromatinmodifying drugs cause cell death in human and canine osteosarcoma cell lines. PLOS ONE 7, e43720 (2012).

23. Blattmann, C. et al. Histone deacetylase inhibition sensitizes osteosarcoma to heavy ion radiotherapy. Radiat. Oncol. 10, 146 (2015).

24. Luo, J., Su, F., Chen, D., Shiloh, A. \& Gu, W. Deacetylation of p53 modulates its effect on cell growth and apoptosis. Nature 408, 377-381 (2000).

25. Stankov, M. V. et al. Histone deacetylase inhibitors induce apoptosis in myeloid

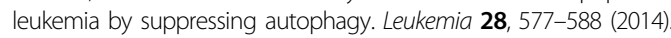

26. Zhang, J. et al. Histone deacetylase inhibitors induce autophagy through FOXO1-dependent pathways. Autophagy 11, 629-642 (2015).

27. Di Pompo, G. et al. Novel histone deacetylase inhibitors induce growth arrest, apoptosis, and differentiation in sarcoma cancer stem cells. J. Med. Chem. $\mathbf{5 8}$ 4073-4079 (2015)

28. Bauer, S. et al. Phase I study of panobinostat and imatinib in patients with treatment-refractory metastatic gastrointestinal stromal tumors. Br. J. Cancer 110, 1155-1162 (2014)

29. Vitfell-Rasmussen, J. et al. A phase $1 / / l$ clinical trial of belinostat (PXD101) in combination with doxorubicin in patients with soft tissue sarcomas. Sarcoma 2016, 2090271 (2016).

30. Cassier, P. A. et al. A phase II trial of panobinostat in patients with advanced pretreated soft tissue sarcoma. A study from the French Sarcoma Group. Br. J. Cancer 109, 909-914 (2013).

31. Furlan, A. et al. Pharmacokinetics, safety and inducible cytokine responses during a phase 1 trial of the oral histone deacetylase inhibitor ITF2357 (givinostat). Mol. Med. 17, 353-362 (2011).

32. Rambaldi, A. et al. A pilot study of the histone-deacetylase inhibitor givinostat in patients with JAK2V617F positive chronic myeloproliferative neoplasms. Br. J. Haematol. 150, 446-455 (2010).

33. Zappasodi, R. et al. Pleiotropic antitumor effects of the pan-HDAC inhibitor ITF2357 against C-Myc-overexpressing human B-cell non-Hodgkin lymphomas. Int. J. Cancer 135, 2034-2045 (2014).

34. Golay, J. et al. The histone deacetylase inhibitor ITF2357 has anti-leukemic activity in vitro and in vivo and inhibits IL-6 and VEGF production by stromal cells. Leukemia 21, 1892-1900 (2007).

35. Todoerti, K. et al. Pleiotropic anti-myeloma activity of ITF2357: inhibition of interleukin-6 receptor signaling and repression of miR-19a and miR-19b. Haematologica 95, 260-269 (2010). 
36. Angeletti, F. et al. Inhibition of the autophagy pathway synergistically potentiates the cytotoxic activity of givinostat (ITF2357) on human glioblastoma cancer stem cells. Front. Mol. Neurosci. 9, 107 (2016).

37. Del Bufalo, D. et al. Histone deacetylase inhibition synergistically enhances pemetrexed cytotoxicity through induction of apoptosis and autophagy in non-small cell lung cancer. Mol. Cancer 13, 230 (2014).

38. Klionsky, D. J. et al. Guidelines for the use and interpretation of assays for monitoring autophagy (3rd edition). Autophagy 12, 1-222 (2016).

39. Coomans de Brachene, A. \& Demoulin, J. B. FOXO transcription factors in cancer development and therapy. Cell. Mol. Life Sci. 73, 1159-1172 (2016).

40. Rodrigues, M., Kalinowska, W., Zielinski, C. \& Sinzinger, H. Verapamil decreases accumulation of $99 \mathrm{Tcm}-\mathrm{MIBI}$ and $99 \mathrm{Tcm}$-tetrofosmin in human breas cancer and soft tissue sarcoma cell lines. Nucl. Med. Commun. 22, 645-650 (2001).

41. Pistritto, G., Trisciuoglio, D., Ceci, C., Garufi, A. \& D’Orazi, G. Apoptosis as anticancer mechanism: function and dysfunction of its modulators and targeted therapeutic strategies. Aging 8, 603-619 (2016)
42. Ferraiuolo, M., Di Agostino, S., Blandino, G. \& Strano, S. Oncogenic intra-p53 family member interactions in human cancers. Front. Oncol. 6, 77 (2016).

43. Koeneke, E., Witt, O. \& Oehme, I. HDAC family members intertwined in the regulation of autophagy: a druggable vulnerability in aggressive tumor entities. Cells 4, 135-168 (2015).

44. Fulda, S. \& Kogel, D. Cell death by autophagy: emerging molecular mechanisms and implications for cancer therapy. Oncogene 34, 5105-5113 (2015).

45. Schmitt-Ney, M. \& Camussi, G. The PAX3-FOXO1 fusion protein present in rhabdomyosarcoma interferes with normal FOXO activity and the TGF-beta pathway. PLOS ONE 10, e0121474 (2015).

46. Guan, H. et al. FOXO1 inhibits osteosarcoma oncogenesis via Wnt/betacatenin pathway suppression. Oncogenesis 4, e166 (2015).

47. Lopez, G. et al. Combining PCl-24781, a novel histone deacetylase inhibitor with chemotherapy for the treatment of soft tissue sarcoma. Clin. Cancer Res. 15, 3472-3483 (2009).

48. Ragazzoni, Y. et al. The thiazole derivative CPTH6 impairs autophagy. Cell Death Dis. 4, e524 (2013). 